\title{
EL ROL DE LA REVISTA GEOLÓGICA DE AMÉRICA CENTRAL EN LA DIFUSIÓN DE VULCANOLOGÍA ENTRE 1984 Y EL 2014
}

\author{
THE ROLE OF THE CENTRAL AMERICAN JOURNAL O FEOLOGY IN THE \\ DIFUSSION OF VOLCANOLOGY BETWEEN 1984 AND 2014
}

\author{
Guillermo E. Alvarado \\ Red Sismológica Nacional y Centro de Investigaciones Geológicas, \\ Universidad de Costa Rica \\ Instituto Costarricense de Electricidad. Área de Amenazas y Auscultación \\ Sísmológica y Volcánica, Apdo. 10032-1000, COSTA RICA \\ galvaradoi@ice.go.cr
}

(Recibido: 26/05/2014; aceptado: 12/08/2014)

\begin{abstract}
Since 1984, 50 issues of the Revista Geológica de América Central (RGAC) have been published, including a special unnumbered volume, with the $50^{\text {th }}$ issue published in 2014 . The most common topics addressed among all articles include volcanic stratigraphy, petrology, geomorphology and volcanic monitoring. Volcanology-related themes represent $22 \%$ of all papers published in RGAC; over 30 years, there have been 86 such papers published, at a rate of 2.8 papers per year. Topics of volcanology are surpassed only by stratigraphy in general, which includes volcanic stratigraphy, radiochronology, and tephrostratigraphy. Since 1994, topics of volcanology have become increasingly common, as noted by several issues with 40 pages or more on related themes. Of the 51 issues of RGAC in 30 years, three were special volumes on Volcanology (No. 30, 2000; No. 43, 2010; and No. 48, 2013). Most published articles are geographically related to Costa Rica (88\%), with very little work from the rest of the countries of Central America and none of Guatemala. While the community is working to encourage volcanological and geological studies in the rest of Central America, a number of constraints, particularly a lack of academic researchers in those countries, makes this a difficult task. The challenge for future generations is to maintain and pursue these goals at a national level.

Keywords: Revista Geológica de América Central, volcanology, papers, historical rol.
\end{abstract}

RESUMEN: Desde 1984 cuando aparece el primer número de la Revista Geológica de América Central (RGAC) hasta el presente $\left(\mathrm{N} .^{\circ} 50,2014\right)$, se han emitido 50 números y un volumen especial sin numeración. Los tópicos de vulcanología que más se repiten en orden preferencial son los de estratigrafía volcánica, petrología, geomorfología y vigilancia volcánica. Los trabajos de vulcanología representan un $22 \%$ del total de trabajos presentados en la RGAC 
y en 30 años se han publicado 86 trabajos a una razón de 2,8 trabajos por año. Quizás dicha temática solo es superada por los de estratigrafía en general, dado que se le sumarían los de estratigrafía volcánica, tefroestratigrafía y radiocronología. A partir del 2004 se nota un despunte en los trabajos de vulcanología, con la aparición de varios números que contienen 40 páginas o más relacionadas con vulcanología. De los 51 números de la RGAC en 30 años, tres fueron volúmenes especiales sobre Vulcanología $\left(\mathrm{N} .^{\circ} 30,2000 ; \mathrm{N} .^{\circ} 43,2010\right.$ y N. $\left.{ }^{\circ} 48,2013\right)$. La mayoría de los trabajos están relacionados con Costa Rica (88\%), existiendo muy pocos trabajos del resto de los países de América Central y ninguno de Guatemala. La tarea de incentivar publicaciones o notas geológicas sobre vulcanología y en general de geología en el resto de América Central está pendiente. Dicha labor no es fácil de subsanar, debido a una serie de limitantes, en particular de personal académico. El reto para las generaciones futuras es el de mantener y superar estas marcas y metas hasta ahora impuestas a nivel nacional en lo que se refiere a trabajos de vulcanología.

Palabras clave: Revista Geológica de América Central, vulcanología, publicaciones, rol histórico.

\section{INTRODUCCIÓN}

Con el establecimiento de la Revista Geológica de América Central (RGAC) en 1984, se abre la posibilidad para los geólogos centroamericanos y foráneos al istmo, publiquen sus investigaciones, que pueden variar desde estudios muy completos y detallados, de gran trascendencia internacional, hasta notas geológicas, noticias y mapas geológicos. Previo al establecimiento de la RGAC, prácticamente la única revista que publicaba aspectos de geología era el Informe Semestral del Instituto Geográfico Nacional, que estuvo activa desde principios de la década de 1960 hasta inicios de la década de 1980 (posiblemente 1983), justo cuando la RGAC aparece, como una necesidad para llenar este vacío. Paralelamente, existían otras revistas generales o especializadas a nivel nacional, que nacieron relativamente en forma simultánea, en donde también se podía publicar aspectos sobre geología, tales como Geoistmo (Escuela de Historia y Geografía, UCR), Revista de Geografía (Universidad Nacional, UNA), Boletín del Observatorio Vulcanológico del Arenal y Miravalles (OSIVAM, ICE), el Boletín de Vulcanología (OVSICORI, UNA), Vínculos (Museo Nacional de Costa Rica), Tecnología en Marcha (Instituto Tecnológico de Cartago) y Biología Tropical (Escuela de Biología, UCR). De todas ellas, tan solo sobreviven las últimas tres. Sin embargo, tan solo en la reconocida revista Biología Tropical, los geólogos eventualmente publican aspectos geológicos relacionados con áreas biogeográficas de gran interés naturalístico.

Resulta bien conocido que publicar en revistas internacionales de clase $\mathrm{A}$, posee una serie de limitaciones y ventajas hacia la contribución del desarrollo del bagaje del conocimiento de una determinada ciencia o tecnología. Dentro de las limitaciones, los artículos deben de ser escritos en inglés (lengua no materna para los centroamericanos), de una forma no solo gramaticalmente correcta, sino en su estilo y forma de presentación. Esta situación muchas no es fácilmente alcanzable por quienes se han especializado en países latino parlantes o incluso en países con otros idiomas. Por otro lado, en estas revistas tan especializadas, por lo general no se puede publicar aspectos de interés para una población profesional y técnica amplia, que tengan implicaciones para la vida cotidiana. En nuestro caso, como geólogos, se refiere a los estudios locales y mapas geológicos, que son de uso cotidiano y consulta por los profesionales en geociencias, pero también por otros profesionales que buscan allí aspectos complementarios para su investigación (recursos naturales, hidrogeología, amenazas naturales, planificación territorial, protección de acuíferos, estado actual de los volcanes y de la sismicidad, etc.). En la otra mano, al publicar en revistas internacionales de gran prestigio, se dan a conocer los logros nacionales en ciencias y se pone al país en "el mapa" internacional, con un gran prestigio no solo para el autor y la institución que representa, sino para la propia región. 
El presente artículo tratará de revisar de manera específica la contribución que ha tenido la RGAC en la Vulcanología a nivel de América Central y el mundo en general. Se define Vulcanología (o Volcanología) como aquella disciplina de la Geología, Geofísica o Geoquímica que estudia científicamente los fenómenos volcánicos, transporte y erupción del magma, así como todos sus aspectos relacionados, tanto en volcanes activos, dormidos o incluso extintos o los restos de ellos. Tiene como fin una inmediata aplicación a la vigilancia, la prevención de las erupciones y la disminución de sus efectos, así como el aprovechamiento de los recursos naturales asociados con el vulcanismo, como la energía geotérmica, los recursos minerales metálicos y no metálicos. Además, nos ayuda a comprender la historia geológica y el paisaje que nos rodea.

Dentro de esta definición amplia, vamos a incluir en el presente apartado los estudios que han salido en la RGAC sobre petrología, petrografía y geoquímica de rocas volcánicas, estratigrafía volcánica, incluyendo el caso particular de tefroestratigrafía, geomorfología volcánica y de vigilancia de volcanes (geoquímica, geodesia, termometría, observación visual y particularmente, sismología volcánica).

También se comentará brevemente sobre otras contribuciones a nivel internacional, no solo por que han tenido su impacto en la comunidad científica, sino porque allí se citan muchos de los trabajos publicados en la RGAC.

\section{PUBLICACIONES DE LA RGAC DE 1984 AL 2014}

\section{Temática y región}

No resulta fácil muchas veces encasillar a las diferentes publicaciones dentro de temas específicos, pero se trató en la medida de lo posible para tener una idea. Desde que se estableció la RGAC en 1984, se han publicado una gran cantidad de artículos, en su mayoría relacionados con estratigrafía volcánica y geocronología, incluyendo tefroestratigrafía, seguido por geomorfología volcánica, y por petrología (incluyendo petrografía y geoquímica de rocas volcánicas). La geoquímica de gases se puso por aparte de la vigilancia volcánica, dado que en su mayoría corresponde con resultados de campañas, más enfocadas a obtener informaciones puntuales, que hacia una vigilancia sistemática y continua con fines de pronóstico; lo anterior es válido para los pocos estudios geofísicos (Cuadro 1). Se desglosan los estudios enfocados a vigilancia volcánica y a actividad eruptiva. Los informes de la RSN (16 en total) corresponden con los reportes de la actividad anual en Costa Rica bajo el título: "Resumen de la actividad sísmica y volcánica de Costa Rica". Desgraciadamente, a criterio del autor, estos informes aportan poco al conocimiento dado que poseen datos muy generales, contrario a los informes extensos y detallados emitidos por la RSN, o aquellos cristalizados en publicaciones.

La RGAC ha capitalizado el número de trabajos (76 publicaciones) que tratan sobre la vulcanología en Costa Rica (88 \%). Sobresale y sorprende los pocos trabajos que hay sobre temas en vulcanología en el resto de los países centroamericanos, estando en tal caso Nicaragua a la vanguardia con 5 trabajos, en su mayoría estudios realizados por foráneos, pero muy lejos del grado de actividad en publicaciones con respecto a Costa Rica. La gran ausencia es Guatemala, que pese poseer gran cantidad de volcanes activos y dormidos, no presenta una sola publicación en la RGAM que tenga que ver con vulcanismo (Cuadro 1). La razón de lo anterior es múltiple y compleja para el resto de América Central, pero se puede resumen en el poco personal en los campos de vulcanología, la poca tradición de publicar y la ausencia de motivación de las instituciones en dicho sentido. Un trabajo se presenta sobre la estratigrafía y las mineralizaciones de un complejo de domos volcánicos en República Dominicana.

Tres volúmenes especiales de la RGAC sobresalen por estar enfocados directamente a vulcanología, lo cual refleja el despunte de la vulcanología dentro del área y la región en general a partirdel 2004. 
Cuadro 1

Publicaciones por temas y países en la RGAC (1984-2004)

\begin{tabular}{cccccc}
\hline Tema & Costa Rica & Panamá & Nicaragua & El Salvador & Otros* \\
\hline Estratigrafía & 23 & & 1 & 1 & 1 \\
Tefroestratigrafía & 7 & 1 & 2 & 1 & \\
Petrología, Petrografía, & 7 & & 1 & & \\
geoquímia de rocas & & & & \\
Geomorfología & 9 & & & \\
Geofísica & 2 & & & \\
Fuentes Termales & 4 & & & \\
Alteración hidrotermal & 2 & & & \\
Sismología & 2 & & & \\
Geoquímica de gases & 5 & & & \\
Peligro volcánico & 3 & & & \\
Vigilancia volcánica & 3 & & & \\
Otros (reseñas, & 8 & & & \\
introducciones, etc.) & 1 & & & \\
Actividad eruptiva & 76 & & & \\
Subtotal de publicaciones & 76 & & & \\
Total de Publicaciones & 86 & & & \\
+Inf. RSN & 16 & & & \\
Gran total & 102 & & & & \\
\hline
\end{tabular}

* República Dominicana

El primero titulado "La Vulcanología y su entorno geoambiental" (Soto \& Alvarado, 2004, editores), abarca diversos temas que abarcan la geovulcanología y morfología volcánica, la estratigrafía volcánica, los depósitos epiclásticos, geoquímica, vigilancia volcánica, la restricción del uso de la tierra en el volcán Arenal y un mapa geológico de un área volcánica. Dicho volumen fue dedicado al vulcanólogo Sergio Paniagua (exdirector de la Escuela Centroamericana de Geología y fundador de la Revista Geológica de América Central), al Dr. Siegried Kussmaul, exdirector de la Revista Geológica de América Central y propulsor de la misma, al geomorfólogo Rodolfo Madrigal (exdirector de la Escuela Centroamericana de Geología) y al Geól. Gregorio Escalante (Fig. 1).
Un segundo volumen especial trató sobre el volcán Poás (Mora-Amador \& Alvarado, 2010, editores). Igualmente abarcó muchos temas, entre ellos neotectónica, geoquímica, sedimentología y tefroestratigrafía, geoquímica de gases y percepción del riesgo.

El tercer y último volumen especial de vulcanología, tuvo por tema el volcán Irazú (Alvarado, 2013 , editor). Incluye temas sobre tefroestratigrafía, análisis la teoría de fragmentación y transporte secuencial, geomorfología y neotectónica, la seudoerupción de 1994, una cueva muy particular con gran cantidad de minerales nuevos dentro del tema de la espeleovulcanología, así como aspectos geoturísticos (El Parque Nacional Volcán Irazú y el Museo de los volcanes Nochebuena). 


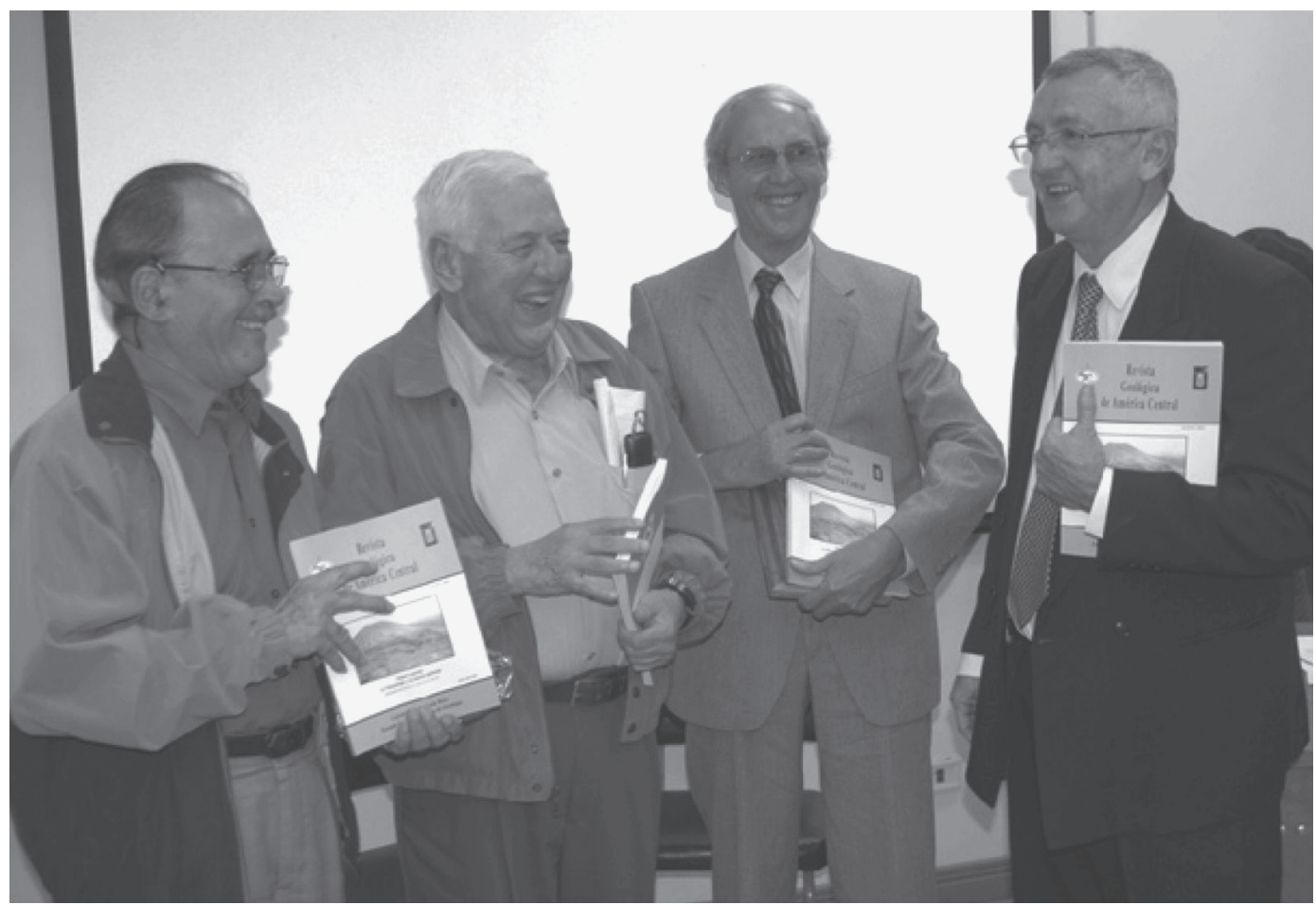

Fig. 1: El primer volumen especial de vulcanología fue dedicado en el 2004 a los geólogos Rodolfo Madrigal, Gregorio Escalante, Siegfried Kussmaul y Sergio Paniagua, por sus cualidades profesionales y académicas, así como por sus contribuciones pioneras en el campo de la vulcanología (tomado de: Soto \& Alvarado, 2006).

\section{Publicaciones por volumen y en el tiempo}

De los siete volúmenes especiales que ha sacado la RGAC, tres de ellos corresponden a vulcanología y con excepción del "Volumen especial Terremoto de Limón 22 de abril de 1991" que posee 223 páginas, todos los volúmenes de vulcanología prácticamente igualan o superan las dos centenares de páginas (el número N. ${ }^{\circ} 48$ alcanzó 199 páginas técnico-científicas, sin tomar en cuenta las de cómo hacer citas bibliográficas). Otro número grande con 200 páginas fue el N. ${ }^{\circ} 7$ (1987), que no corresponde con un volumen especial.

Los números de la RGAC que poseen 40 páginas o más relacionadas con vulcanología, corresponden a: N. ${ }^{\circ} 17$ (1994), N. ${ }^{\circ} 30$ (2004), N. ${ }^{\circ} 33$ (2005), N. ${ }^{\circ} 40$ (2009), N. ${ }^{\circ} 43$ (2010), 46 (2012), N. ${ }^{\circ} 47$ (2012) y N. ${ }^{\circ} 48$ (2013).
Durante estos 30 años (1984-2014), la proporción de publicaciones de vulcanología (número de páginas de los artículos y de los informes de la RSN) con respecto al resto de las temáticas (suma total de las páginas en cada número) es de un $22 \%$. En los 30 años de la RGAC, se han publicado 86 trabajos (excluyendo los informes cortos de la RSN) relacionados con vulcanología ( 2,8 trabajos/año). Aunque esta relación cercana a 3 trabajos por año (o un trabajo relacionado con vulcanismo por cada 5 publicaciones) es muy buena, es relativa, dado que han habido años en donde no se han publicado ningún trabajo científico relacionado con vulcanología (p.ej. N. ${ }^{\circ} \mathrm{s} 4$ y 5 de 1986; N. ${ }^{\circ} 7,1987$, N. ${ }^{\circ}$ s 10 y 11 de 1989 y 1990, respectivamente; N. ${ }^{\circ}$ s 15, 1993; Volumen especial del Terremoto de Limón en 1994; N. 23 del $2000, \mathrm{~N}^{\circ}{ }^{\circ} \mathrm{s} 27$ y 28 del 2002 y $2003 ; \mathrm{N} .{ }^{\circ}$ s 36 , 
39, 42 y 44 del 2007, 2008, 2010 y 2011). Ello quiere decir que los trabajos son relativamente "cíclicos" con despuntes en ciertos años (2004, 2010 y 2013) en función de iniciativas particulares (Fig. 2).

\section{Publicaciones por autor y nacionalidad}

A nivel nacional, el que más ha publicado en la RGAC temas de vulcanología es el suscrito del presente artículo (39 trabajos), seguido por los colegas Gerardo Soto y Esteban Gazel (empatados ambos con 6 trabajos). A nivel internacional, el liderazgo lo mantiene el vulcanólogo italiano Sergio Chiesa (6 trabajos), a pesar a que desde el 2005 no volvió a publicar en la RGAC y que ahora disfrute de su merecida pensión (Fig. 3).

Un aspecto relevante en el campo de la vulcanología de la RGAC, con respecto a otras revistas, es que varios estudiantes realizaron su primera publicación o ensayo (fecha de envío previo) próximos a graduarse o incorporarse al Colegio de Geólogos. Estas se presentan, en el campo de la vulcanología, particularmente a partir del 2004 con 6 artículos que tuvieron la participación de 11 estudiantes, en coautoría con profesionales. Del mismo modo, contemporáneamente, varias publicaciones nacieron basadas en los temas de tesis en vulcanología.

\section{Portadas}

A partir del N. ${ }^{\circ} 23$ (2000) y como caso excepcional el volumen especial del terremoto de Limón (1994), la RGAC presenta su portada con una fotografía alusiva a algún tema geológico. De las 28 portadas de la RGAC que poseen fotografías, al menos $12(43 \%)$ presentan temas afines con la vulcanología o rocas volcánicas, entre ellos: Modelo digital del fondo oceánico con los volcanes submarinos y las cordilleras volcánicas de Costa Rica, rocas volcánicas (los crestones, basaltos del río Lempa, dacitas de Carbonal, rocas volcánicas falladas en Montes de Oro, ignimbritas fallas en El Salvador, dos secciones delgadas de rocas volcánicas), perfil geológico antiguo con los volcanes Irazú y Turrialba y fotografías de los volcanes Rincón de la Vieja y Poás, así como la cueva de los minerales en el volcán Irazú.

\section{CONCLUSIONES Y RECOMENDACIONES}

Claramente al observarse los listados de trabajos, se desprende que en los últimos 30 años, los artículos relacionados con vulcanología superan a los de otras áreas en número y cantidad de páginas. Quizás tan solo van a ser superados por trabajos de estratigrafía general, dado que las

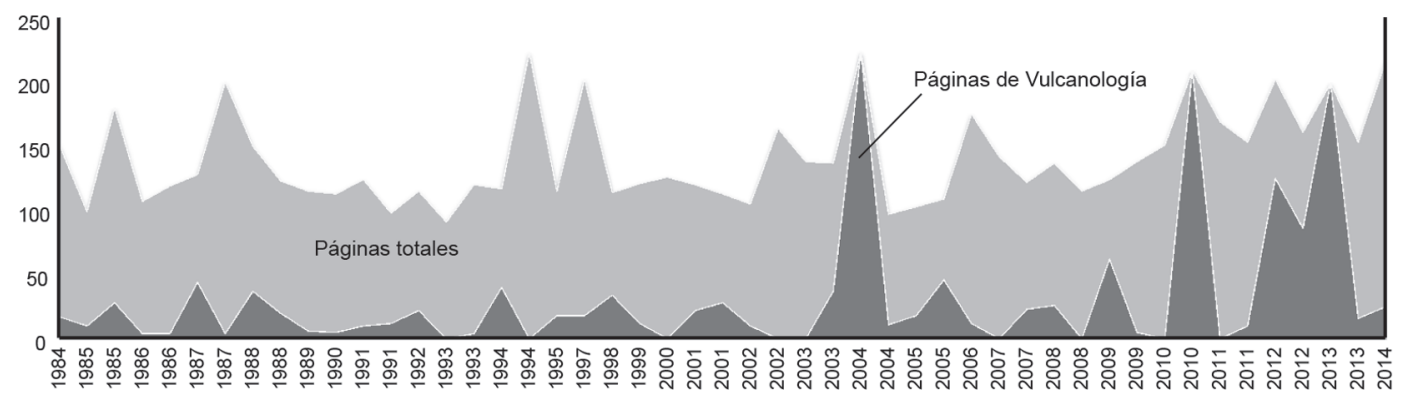

Fig. 2: Número de páginas total de los diferentes artículos en la RGAC de 1984 al 2004 versus el número de páginas de artículos relacionados con el vulcanismo en cada año. Los años repetidos corresponden a la publicación de dos revistas anuales. 

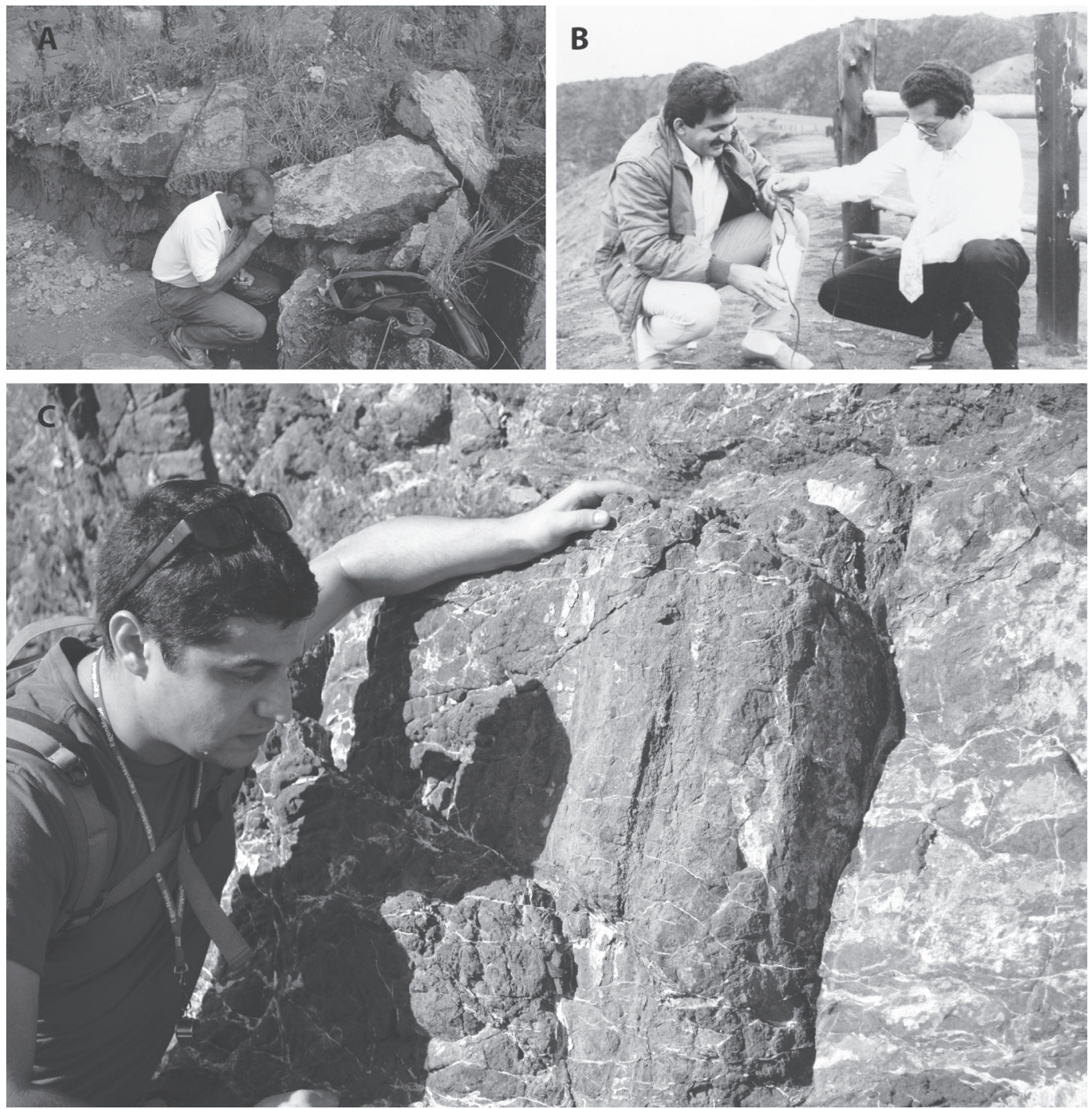

Fig. 3: Fotografías de los vulcanólogos. A) Sergio Chiesa, en pleno trabajo de campo en Guanacaste (1986). B) Gerardo Soto, con corbata, en pleno trabajo de campo en el Irazú (1992) junto con su asistente, Francisco Arias, debido a una alerta volcánica. C) Esteban Gazel en pleno trabajo de campo (2013, fotografía Percy Denyer).

contribuciones de estratigrafía volcánica, tefroestratigrafía y cronología ígnea se pueden sumar a los de estratigrafía de rocas sedimentarias.

Queda como tarea el tratar de fomentar que se publiquen trabajos sobre diversos tópicos de vulcanología en los vecinos países centroamericanos y que sean realizados por ellos.
Un despunte en los trabajo de vulcanología se observa en particular con los tres volúmenes especiales de vulcanología en los números 30 (2004), 43 (2010) y 48 (2013). Queda para las futuras generaciones que en la actualidad se están desarrollando en el tema, el superar esta marca y metas impuestas. 


\section{AGRADECIMIENTOS}

A S. Paniagua y S. Kussmaul, quienes no solo crearon la RGAC sino que motivaron a que los resultados de investigaciones fueran dados a conocer, además de fomentar a que se realizaran diversas investigaciones dentro del ámbito de la Vulcanología. A P. Denyer, quien ha continuado con la labor titánica y muchas veces no reconocida en su verdadera magnitud. No es nada fácil el batallar con los autores para que cumplan con las normas, y el publicar a tiempo la revista. Claramente, la Universidad de Costa Rica también ha tenido un papel protagónico para que todo lo anterior se lleve felizmente a cabo. Muchos estudiantes, hoy día colegas, dieron su aporte a la RGAC, poniendo en evidencia que aún a nivel de estudiante avanzado, resulta posible el contribuir con un grano de arena a la geología y particularmente la vulcanología. D. Szymanski revisó el resumen en inglés.

\section{REFERENCIAS BIBLIOGRÁFICAS}

ALVARADO, G.E. \& SOTO, G.J. (eds., 2004): Número especial: La Vulcanología y su entorno geoambiental.- Rev. Geol. Amér. Central, 30: 1-223.

ALVARADO, G. E. (ed., 2013): Volumen Especial: Volcán Irazú.- Rev. Geol. Amér. Central, 48: 1-227.

MORA-AMADOR, R. \& ALVARADO, G. E. (eds., 2010): Volumen especial: Volcán Poás.- Rev. Geol. Amér. Central, 43: 1-235.

SOTO, G. J. \& ALVARADO, G. E., 2006: In Memóriam de Gregorio Escalante Montealegre (1932-2006).- Rev. Geol. Amér. Central, 34-35: 7-11. 